\title{
Lidil
}

Revue de linguistique et de didactique des langues

$62 \mid 2020$

Recherches actuelles en didactique du lexique : avancées, réflexions, méthodes

\section{Regards sur un parcours de chercheur en didactique du lexique : Francis Grossmann}

Ophélie Tremblay

\section{OpenEdition}

\section{Journals}

Édition électronique

URL : http://journals.openedition.org/lidil/8153

DOI : $10.4000 /$ lidil.8153

ISSN : 1960-6052

Éditeur

UGA Éditions/Université Grenoble Alpes

Édition imprimée

ISBN : 978-2-37747-226-0

ISSN : $1146-6480$

Référence électronique

Ophélie Tremblay, «Regards sur un parcours de chercheur en didactique du lexique

Francis Grossmann », Lidil [En ligne], 62 | 2020, mis en ligne le 03 novembre 2020, consulté le 05 novembre 2020. URL : http://journals.openedition.org/lidil/8153 ; DOI : https://doi.org/10.4000/lidil. 8153

Ce document a été généré automatiquement le 5 novembre 2020

(C) Lidil 


\title{
Regards sur un parcours de chercheur en didactique du lexique : Francis Grossmann
}

\author{
Ophélie Tremblay
}

\section{Introduction}

1 Ce texte présente, à travers des extraits d'un entretien réalisé à l'automne 2017, un aperçu du parcours de chercheur de Francis Grossmann, professeur à l'Université Grenoble Alpes pendant plus de 15 ans et maintenant à la retraite. La restitution de certains extraits de notre entretien permettra, je l'espère, de fournir quelques éléments éclairant le parcours de ce chercheur, notamment dans le champ de la didactique du lexique.

\section{La question du sens au cœur d'un parcours professionnel}

\begin{abstract}
avant d'entrer dans la vie professionnelle, est-ce que tu t'intéressais au VOCABULAIRE?

Initialement, c'était plutôt l'écriture qui m'intéressait, pas particulièrement le vocabulaire, mais forcément c'est lié, parce qu'on écrit avec des mots... C'était plutôt cette dimension d'écriture qui m'intéressait quand j'étais jeune. J'ai écrit beaucoup de poésie, de textes, de choses comme ça. J'ai toujours bien aimé écrire, même après, les dissertations, ça me plaisait beaucoup. Que ce soit d'ordre poétique, ou de l'ordre du construire. Alors les mots là-dedans, oui. J'avais une mère avec qui je discutais beaucoup et on définissait beaucoup les choses. Il ne s'agissait pas forcément de définir les mots en tant que tels, mais plutôt de définir des notions, de se mettre d'accord sur le sens des termes. Alors ce travail méta, il était très présent, ma mère, elle avait une formation philosophique au départ donc c'était plus cette dimension-là qui était forte dans mon enfance et ma prime jeunesse, on va dire. [Rires]
\end{abstract}




\section{COMMENT ALORS S'EST DÉVELOPPÉE TON INCURSION DANS LA DIDACTIQUE DU LEXIQUE ? QU'EST- CE QUI T'A AMENÉ LÀ ?}

[...] mon premier travail universitaire [c'était] avec Robert Martin. Un travail de maitrise. J'étais très intimidé... Quand je pense maintenant à nos étudiants, qui nous voient trente-six fois par jour... Je crois que je l'ai vu deux fois dans l'année! C'était comme ça à l'époque. C'était un mandarin! [Rires] Mais un mandarin sympathique! [Rires] ... Et un maitre. Pour moi ça a été vraiment un maitre. Je pense qu'à l'université, c'est celui qui, parmi mes professeurs, m'a vraiment marqué. Et au moment où j'ai voulu faire ma maitrise, j'ai eu l'idée de m'intéresser à ce qu'on appelait «les textes libres ». C'est un concept qui vient de Célestin Freinet, c'est-à-dire des textes écrits spontanément - entre guillemets, parce qu'en fait il y a toute une démarche pour qu'ils arrivent à les produire -, et donc j'ai proposé ce corpus de textes libres d'enfants, je crois que c'était CE2-CM1. Il [Robert Martin] a été tout de suite d'accord, et sur son conseil j'ai appliqué la méthode des isotopies greimassiennes - puisque c'était aussi la mode de la sémantique structurale - à l'étude des textes d'enfants de 10-11 ans. Et j'ai trouvé ça passionnant. Parce que ces textes étaient déjà, en eux-mêmes, vraiment très intéressants. Mais le problème que j'ai eu c'est que la méthode, finalement, de cette sémantique structurale, n'éclairait pas grand-chose... Robert Martin voulait que je voie les redondances sémiques, bon j'ai fait ça, j'ai essayé de le faire, mais j'ai trouvé ça pas très intéressant, pour expliquer, finalement, la dynamique d'écriture, même au plan lexical, qu'est-ce qui se passait? Donc c'était à la fois très intéressant et très frustrant. Et je pense que c'est la première fois où j'ai marié ce que j'allais essayer de faire pendant une bonne partie de ma vie, c'est-à-dire cet intérêt pour la linguistique - parce que fondamentalement, quand même, il y avait cet intérêt-là-, et puis, la dimension, je dirais pas didactique, parce que c'était pas vraiment didactique, il ne s'agissait pas de mettre en place des démarches didactiques, mais de comprendre comment des enfants de cet âge-là écrivaient, quelle était la logique. Je crois que j'avais appelé ça, très prétentieusement, "la logique des possibles narratifs" ou un truc comme ça... Il s'agissait de comprendre comment ça, ça fonctionnait. Voilà, donc c'est le premier travail.

Après, il s'est trouvé que je suis allé à Strasbourg et j'ai eu d'autres professeurs, j'ai oublié un peu la linguistique, je l'ai oubliée parce que je préparais l'agrégation pour avoir du travail, il fallait que je devienne professeur. J'avais passé le concours des IPES, donc j'étais sur des rails. J'étais financé pour devenir professeur, et j'ai passé le concours, je suis devenu professeur en collège et en lycée. Bien plus tard, il y a eu une période qui a aussi été très utile pour moi, j'étais déjà plus tout jeune, je suis devenu formateur dans une école normale. Ce qui est devenu ensuite les IUFM (puis ESPE, puis INSPE, cela n'arrête pas de changer). Donc, j'ai fait ça plusieurs années. Ça a été extrêmement instructif parce que là, effectivement je me suis intéressé à la didactique. Forcément parce que c'était mon corps de métier, j'ai appris énormément de choses et là, effectivement, la didactique du lexique a été un élément important pour moi.

\section{COMMENT C'EST ARRIVÉ ?}

Comment c'est arrivé... C'était toujours cette envie de relier cet intérêt pour la linguistique et notamment aussi pour la sémantique qui était aussi ma passion depuis mes plus jeunes années d'étudiant. Euh... Donc, une sémantique qui soit une sémantique qui ne soit pas une sémantique purement formelle - même si j'étais aussi fasciné par la sémantique formelle-, mais plutôt la sémantique en tant que fondement des pratiques langagières; la sémantique lexicale et même la sémantique grammaticale m'intéressaient beaucoup dans la mesure où elles donnent des fondements et elles permettent de voir le langage à travers cette question du 
sens, qui finalement est mis de côté par tous les courants formalistes. Donc, ça, ça m'a beaucoup intéressé, mais ce qui m'a aussi intéressé c'est le fait que... et ça, je crois qu'on l'a vraiment développé et toutes sortes de chercheurs l'ont développé, ce lien entre le lexique et les énoncés, le discours. Donc le lien entre les sens des mots, mais aussi leur organisation, leur intégration dans d'autres plans. Le plan syntaxique, bien sûr, et le plan énonciatif ... ça a pris de plus en plus d'importance, cet aspect-là, pour moi, même si c'est parfois difficile, l'organisation du discours. Finalement, je ne suis pas sorti de mes textes d'enfants du tout début, puisque c'était déjà ça qui m'intéressait. Même si je n'avais pas trouvé la solution, à l'époque, avec les isotopies greimasiennes. [Rires]

\section{Des projets de recherche structurants}

\section{PEUX-TU ME PARLER DES PROJETS IMPORTANTS QUI ONT ORIENTÉ tON PARCOURS EN RECHERCHE?}

Si je me cantonne aux recherches collectives, je m'étais intéressé, d'abord, aux pratiques de lectures étudiantes, au sein de la petite équipe qu'avait formée Michel Dabène, avec entre autres Cathy Frier, Françoise Boch, Jean-Pascal Simon et Marie-Cécile Guernier. Michel avait su créer un climat de confiance et de travail d'équipe formidable. Nous n'étions pas centrés particulièrement sur le lexique, c'était la notion de "représentation verbalisée" comme moyen d'accès à la compréhension / interprétation des textes qui servait de fil conducteur à la recherche. Et puis après, il y a eu un projet intéressant avec Cathy Frier, qui reprenait la question des lectures partagées, que j'avais explorée dans ma thèse. Nous avons creusé ensemble la question des modes de lectures des albums, telle qu'elle était faite en milieu familial. Là encore, le lexique n'était présent qu'en arrière-plan, à travers la question des formes de reformulation et d'explicitation lexicale, deux aspects que j'avais déjà commencé à travailler, et dont je rends compte dans le bouquin Enfances de la lecture. En ce qui concerne plus directement le lexique, il y a eu un projet qui s'appelait "École et sciences cognitives", que j'ai copiloté avec un collègue, Georges Antoniadis, et on a collaboré avec des psychologues et des collègues qui étaient dans d'autres cadres théoriques. Ce qu'il y avait d'intéressant à Grenoble, c'est qu'on avait pu mettre en place une collaboration entre des gens qui s'entraidaient et se stimulaient intellectuellement. Des collègues avec qui je collaborais de longue date, comme Françoise Boch ou Cathy Frier, mais aussi des collègues nouvellement arrivées, comme Agnès Tutin, qui nous a mis sur la piste des collocations, un domaine d'exploration qui a été essentiel et qui nous a orientés vers la théorie Sens-Texte, tout cet univers issu de l'Université de Montréal qu'on ne connaissait pas. [...]. On venait aussi de recruter Cristelle Cavalla qui était toute nouvelle chez nous, mais pleine de bonne volonté, avec un dynamisme formidable. Donc, je suis retourné dans les classes avec elle, ce que je n'avais plus fait depuis ma période en IUFM, je m'en souviens, ç'a été une expérience mémorable.

TU M'AS dÉJÀ EXPLIQUÉ QUE dANS CE PROJET, VOUS AVIEZ PRIS CONSCIENCE QUE LA PRISE EN Compte des Émotions du personnage ne passe pas tellement par les mots QUi DÉSIgNENT LES ÉMOTIONS EUX-MÊMES, MAIS PAR LA FAÇON dE DÉCRIRE COMMENT CELLES-CI SE TRADUISENT ET SE MANIFESTENT AU FIL DU TEXTE.

Oui, on a été confrontés à ce problème, didactiquement, on a essayé de résoudre ce petit problème en tenant notre bout, qui était le lexique des émotions, en partant de la catégorisation, comment on catégorise les émotions. Mais il y a un fossé entre cette question de la catégorisation et la manière dont un scripteur, finalement, traduit les émotions, et c'est aussi intéressant de savoir à quel moment, quand on écrit une histoire, on a besoin de traduire les émotions du personnage. Donc voilà, c'est entre autres, ce problème didactique qui nous 
avait intéressés, sans que nous perdions pour autant l'intérêt sur les questions liées au lexique des émotions proprement dit. Alors évidemment, le projet a été très utile parce qu'il nous a fait beaucoup avancer au plan linguistique tout comme au plan didactique, on a travaillé vraiment avec Agnès, Françoise, Cristelle, et ... on était toute une tripotée à travailler sur le lexique des émotions. [Rires]

Ç'a été un beau projet. Je pense qu'il n'a pas été complètement réussi en raison de difficultés de compréhension avec les autres équipes. [...] je pense qu'il nous a manqué un vrai séminaire de travail avec les collègues des autres disciplines, dans lequel on aurait explicité chacun nos positions pour voir à partir d'un état des lieux qui aurait été fait de manière réfléchie comment on pouvait construire un objet commun. Mais comme toujours, il fallait construire dans l'urgence quelque chose qui convienne aux décideurs, qui soit dans l'air du temps où il y a à la fois des didacticiens, des linguistes, des psychologues et des gens qui s'intéressaient plutôt à la dimension outillée. Sur ce plan, on a réussi, ça a plu, mais voilà, c'est un objet un peu artificiel parce qu'il n'était pas suffisamment mûri dans une réflexion collective. Donc moi,j'ai retenu ça et c'est vrai qu'avec Agnès Tutin quand on a porté le projet "Scientex", là, alors c'était différent, parce qu'on était vraiment sur nos propres intérêts de recherche et qu'on savait mieux où on voulait aller. La question des collocations a été centrale pour moi parce qu'elle permettait enfin de sortir la recherche sur le lexique d'une approche centrée sur le mot, en envisageant à la fois le lien avec la syntaxe et le discours, ainsi que celle des genres discursifs scientifiques.

Alors après, la vie du laboratoire a fait qu'il a fallu choisir un axe. L'axe 1, qui est l'axe linguistique, l'axe 2, acquisition et sociolinguistique ou le 3, la didactique. Donc, pour des raisons de structuration du laboratoire, tout à fait légitimes [...], je me suis concentré sur l'axe 1, et j'ai recentré mes recherches en linguistique. Ça m'a beaucoup occupé durant les années qui ont suivi. C'est ce qui explique que le volet didactique a été un petit peu perdu de vue... Ce qu'il faut quand même dire, c'est que même positionné plus clairement en tant que linguiste, j'ai toujours eu le souci, y compris dans l'axe 1, de ce qu'on n'ose pas appeler en France, parce que mal vu, «application». En France, les didacticiens ont horreur du mot. Moi, il ne me dérange pas tant que ça, dans la mesure où je pense que...

\section{C'Étalt UNe des phases de la didActisation, que de faire UNe première proposition D'APPLICATION.}

Voilà. Après, ce qui gêne les didacticiens - y compris, celui qui m'avait formé (au doctorat), c'est-à-dire Michel Dabène-, c'était de dire qu'on va transposer de manière quasiment mécanique des notions linguistiques... Mais là, dès qu'on est un peu intelligent, c'est jamais ça qu'on fait. Par contre, le risque inverse me semble nettement plus grand. C'est de faire de la didactique sans avoir une armature conceptuelle. Alors après, moi, mon idée là-dessus c'est que bien entendu, quand on se situe comme didacticien nourri par la linguistique, comme j'ai essayé de l'être, on sait que ce n'est qu'une partie de la didactique. Il y a des gens qui sont psychologues ou qui viennent d'autres disciplines qui ont tout à fait des choses à dire qu'on ne dit pas. Je trouve que c'est un peu un faux débat que ce refus du terme "application", parce que pour moi, de transpositions mécaniques, il n'en a jamais été question. Je pense que ça vient de l'histoire des arbres syntaxiques où des gens avaient cherché à faire de la syntaxe de manière très...

\section{... " APPLICATIONNISTE " ?}

Oui, mais applicationniste, vraiment: on prend des concepts qui perdent tout sens dans le contexte de la classe. Donc après, on peut se dire, "voilà, on a des concepts en linguistique, 
comment est-ce qu'on peut construire un apprentissage méthodique, par exemple en didactique du lexique ". C'est comme ça qu'on a essayé de le faire. Moi, je suis plutôt là-dedans. Après si quelqu'un me dit "Oui, mais dans votre démarche didactique du lexique, il manque un élément important comme la motivation ", ou je ne sais quoi, on va l'intégrer. Mais ce sont des gens qui sont spécialistes de ces choses-là qui pourront le faire mieux que nous. Voilà, c'est un peu ma manière de voir les choses là-dessus.

\section{$[\ldots]$}

Donc, une approche - ça, je pense que je ne l'ai pas assez creusée dans l'article 1 qui me semble en fait plus rationnelle. Qui ne cherche pas à tout prix à rattacher des signifiés, par l'usage, par l'histoire, par je ne sais quels autres mécanismes... On finit par s'éloigner tellement qu'ils sont maintenant perçus comme d'autres éléments lexicaux. Ils sont plus rattachables à la conscience linguistique qu'à cette fameuse sensibilité comme tu l'as appelée.

\section{... " WORD CONSCIOUSNESS " ?}

Oui, donc voilà... Ça serait ridicule de vouloir à tout prix - surtout avec des enfants ou des jeunes qui sont en train d'apprendre la langue ou avec des étrangers qui apprennent la langue -, à chaque fois, faire une espèce d'histoire culturelle un peu comme le fait parfois Jacqueline Picoche, même si elle a fait beaucoup de choses très utiles didactiquement... On ne peut pas toujours partir d'un signifié de puissance qui permettrait de motiver l'existence de toutes les acceptions d'un mot polysémique. Une telle démarche a des limites, parce que ça a un côté un peu artificiel par moment de vouloir à tout prix retrouver des liens sémantiques... On connecte finalement des choses qui sont un peu déconnectées. Est-ce que c'est toujours utile de reconnecter les choses? Mais, ponctuellement, à certains moments, c'est aussi enrichissant d'avoir cette approche culturelle du lexique.

Donc, si je devais reprendre cet article ou le creuser, ça serait de dire, voilà, qu'est-ce qu'on fait avec ces différentes approches? Parfois, ça peut être intéressant de faire du Picoche. Et, en quoi, à quel moment c'est plus intéressant? Il y a ça, que j'essaierais de creuser et puis peut-être aussi, la dimension français langue étrangère qui n'est pas vraiment ma spécialité, mais qui m'a toujours intéressée... J'avais une étudiante de thèse, Veronica Benigno, qui est italienne, et qui s'était intéressée à ressusciter les fameux vocabulaires de base issus de Gougenheim. Je pense qu'aujourd'hui on pourrait reprendre ce chantier. Je l'ai dit dans l'article, mais c'était très programmatique et maintenant, il y a des travaux. Donc, il faudrait actualiser l'article, en regardant, ce qui se fait vraiment, ce qui s'est fait. Et puis, surtout, ce qu'on pourrait encore faire puisque grâce à toute une série d'outils, on peut maintenant, faire de manière beaucoup plus rapide et approfondie, ce travail de récolte de données que Gougenheim avait fait à l'époque.

Là encore, il faudrait avancer des pistes beaucoup plus concrètes que ce que j'avais raconté en synthétisant. Pour voir comment on peut armer les enseignants, parce que quand on est sur le terrain on n'a pas le temps de fabriquer ce type de chose.

DONC, IL Y A ENCORE, LA QUESTION DE CE TRANSFERT QUI N'EST PLUS DE L'APPLICATION, MAIS VRAIMENT UN TRANSFERT DE CONNAISSANCES.

Qui est la possibilité de développement, de donner des outils pratiques à des enseignants pour qu'ils puissent faire des leçons de vocabulaire ou de lexique de manière intelligente. 


\section{De nouveaux chantiers}

EN TERMINANT, EST-CE QU'Ll Y A UN PROJET QUE TU AURAIS AIMÉ dÉVELOPPER, SI TU L'AVAIS PU, AU fil de TA CARRIĖRE, OU QUE TU dÉVELOPPERAIS DANS LES PROCHAINES ANNÉES ?

Je ne sais pas si je le développerais, mais c'est vrai que, ce qui m'aurait peut-être intéressé aussi, c'était de relier - parce que ça avait été un peu mon travail de thèse avec les institutrices de maternelle -, de travailler sur ces questions de lectures partagées. [...] C'est un chantier que j'ai laissé complètement tomber, pour me plonger dans les collocations. Alors qu'en fait, les deux choses auraient pu être reliées. Donc, je me dis maintenant que je saurais comment relier les deux choses. D'autre part, je pense qu'à l'époque où je travaillais là-dessus, je travaillais aussi sur les albums. C'est aussi un beau chantier. Alors, il est compliqué ce chantier, parce qu'il ne faudrait pas tomber dans une vision trop utilitariste qui ne consisterait à voir les albums pour enfants que comme des réservoirs de mots puisque c'est avant tout, des outils pour une dynamique d'interaction entre l'adulte qui raconte, ou qui lit, et des enfants. Mais, n'empêche que c'est quand même intéressant de voir comment ils sont constitués, quel est ce lexique. Ça a des tas d'implications à la fois en termes de stéréotypes sociaux, en termes de construction du monde, puisque finalement, c'est un des premiers outils qui permet d'arriver à faire voir le monde aux enfants. En fait le monde, qui n'est pas leur monde le plus proche, le monde de l'imaginaire. Ça aussi c'est une chose que je n'ai pas creusée, puisque je suis parti sur d'autres choses notamment l'écrit scientifique.

Aussi, ce qui me frappe, c'est que quand on lit la littérature scientifique, il y a une espèce de leitmotiv obligé qui est que la didactique du lexique est le parent pauvre. On répète ça depuis je ne sais pas combien d'années. Moi-même je l'ai dit, parce que tout le monde le disait! [Rires] On se dit parent pauvre, mais attends... Il est peut-être temps de changer de discours et d'être beaucoup plus... De montrer ce qu'on a fait, ce qui a été fait. Donc, il faut valoriser les travaux, montrer qu'il y a une longue tradition, en fait, en didactique du lexique.

Donc, je pense qu'il faut qu'on arrête avec ce discours "misérabiliste", on va dire. Et puis, proposer des choses enthousiasmantes qui donnent envie aux profs, aux enseignant.e.s, de faire des choses. Il faut aussi avoir cette dimension intégrative. C'est pour ça que ce dialogue aussi entre les approches est important: voir ce qu'il y a de vraiment utile dans tout cet héritage de Jacqueline Picoche, qui est vraiment très riche, qui nous a donné beaucoup de choses. Et puis, les travaux issus plutôt de la tradition, de la linguistique Sens-Texte, de la sémantique des cadres ou d'autres approches, qui ne sont pas du tout des univers irréconciliables. On est sur la même planète lexicale. [Rires]

\section{Conclusion}

Dans cet entretien, Francis Grossmann partage un pan de son histoire personnelle et professionnelle, nous donnant ainsi accès aux rencontres et évènements marquants de son parcours de chercheur. Ce récit illustre la force et le rôle catalyseur des liens humains dans la construction de la vie professionnelle et le développement des champs de recherche. Il montre aussi la part du rapport affectif envers ce qui deviendra un objet d'étude scientifique pour un.e. chercheur.e, ici, l'écriture, les mots et le discours. À travers ce partage, il nous convie, me semble-t-il, à poursuivre le travail et à explorer les pistes qu'il a lancées pour contribuer à notre tour au développement de la didactique du lexique comme champ de recherche et de formation. 


\section{NOTES}

1. Il fait ici référence à l'article suivant: Grossmann, F. (2011). Didactique du lexique : état des lieux et nouvelles orientations. Pratiques, 149-150, 163-183. <https://doi.org/10.4000/pratiques. 1732>.

\section{AUTEUR}

\section{OPHÉLIE TREMBLAY}

Université du Québec à Montréal 